\title{
EDITORIAL
}

\section{Mohammed Bin Khalifa Bin Salman Al Khalifa Cardiac Centre: The Centre of Excellence for Electrophysiology}

\section{Adel Khalifa Sultan Hamad*}

Consultant Cardiologist \& Interventional Cardiac Electrophysiologist, Mohammed bin Khalifa Al Khalifa Cardiac Centre, Awali, Kingdom of Bahrain.

\section{*Corresponding author:}

Adel Khalifa Sultan Hamad, BMS, MD, FGHA, FRCP (Canada), Consultant Cardiologist \& Interventional Cardiac Electrophysiologist, Head of Electrophysiology Department, Mohammed bin Khalifa Al Khalifa Cardiac Centre, Awali, Bahrain. E-mail: dradelkhalifa@yahoo.com. Tel: + (973) 39441888

Received date: 21 July 2021; Accepted date: 10 August 2021; Published date: December 31, 2021

In this article, the author describes the exceptional electrophysiology program at Mohammed Bin Khalifa Bin Salman Al Khalifa Cardiac Centre in Bahrain.

The Mohammed Bin Khalifa Bin Salman Al Khalifa Cardiac Centre was founded in 1992 as an organization inspired by the late His Royal Highness Sh. Khalifa bin Salman Al Khalifa, the Prime Minister of Bahrain (1970-2020). From humble beginnings some three decades ago, Mohammed Bin Khalifa Bin Salman Al Khalifa Cardiac Centre has now grown to become one of the finest and most prestigious tertiary care hospitals not only in Bahrain, but in the region as well, enabled with excellent infrastructure and cutting-edge technology, along with professional expertise. The center began as a small facility with only one inpatient ward for cardiology and cardiac surgery. The primary focus was on coronary interventional procedures and cardiac surgery in general. Nevertheless, there was no electrophysiology (EP) service, and therefore all patients who required arrhythmia ablation or high-voltage implantable cardiac devices were sent abroad. As a result of the devotion and commitment shown by the administration, the center has expanded to include two inpatient wards in addition to a coronary care unit (CCU) and coronary intensive care unit (CICU).
The new Mohammed bin Khalifa bin Salman Al Khalifa Cardiac Center will transform Bahrain's cardiac care facilities to a higher level following its inauguration by His Royal Highness the Crown Prince and Prime Minister Prince Salman bin Hamad Al Khalifa on Friday, February 4, 2021 on behalf of His Majesty King Hamad bin Isa Al Khalifa. Currently the Cardiac Centre has 148 beds in four wards and is located in Awali, a small municipality in Bahrain. Five thousands square meters of spacious, seven-story Cardiac Centre house the EP department. There are areas designated for adults and children, emergency rooms dedicated to cardiac disorders, intensive care, day cases, operating theatres, four high-tech catheterization labs, and a hybrid operating room. In order to cater to the patient's needs, the center strives to foster a caring, compassionate and loving environment.

In 2008, the EP department established its first arrhythmia clinic, which is the first of its kind to deal with arrhythmia assessment and management. Previously, electrophysiology procedures were carried out once a week, but they are now carried out two to four times per week. Among the amenities available at the newly opened center is a dedicated EP lab. It was once a shared asset between EP and interventional cardiology departments. When fully operational, it will enable more procedures to be 
performed. In 2008, the EP department lacked dedicated technicians and had to rely on expats for device implants, EP procedures, and to run the device clinic. Now, there are three Bahraini technicians on the team. The Department gained another consultant electrophysiologist in 2012, which also led to an increase in the volume and complexity of the procedures conducted by the Department. At present, the day-to-day management of the EP service is handled by two electrophysiologists and three technicians. As opposed to 5-10 consultations per week at the device clinic a decade ago, the department now sees around 35 patients weekly, showing that it has grown by leaps and bounds in the past decade.

The EP Services at Mohammed Bin Khalifa Bin Salman Al Khalifa Cardiac Centre implanted Bahrain's first implantable cardioverter defibrillator (ICD) in 2009. In subsequent months, the first Cardiac Resynchronization Therapy (CRT) implant was also performed. Patients requiring these procedures had previously been sent to Kuwait and Jordan. The EP department has additionally established the first cardiac electrophysiology laboratory in Bahrain for arrhythmia diagnosis and ablation and started with Atrioventricular Nodal Reentrant Tachycardias (AVNRTs) and WolffParkinson-White Syndromes (WPW) ablations in 2009. This has been advantageous as people with these conditions had to be sent abroad for treatment.

The EP lab at Mohammed Bin Khalifa Bin Salman Al Khalifa Cardiac Centre is part of the cardiac catheterization labs and has sterile environments for patient preparation, holding, and recovery, as well as support areas such as scrub rooms and control rooms. It is spacious enough for patients to move about, physicians to perform procedures, and EP equipment to be accommodated.

Several EP therapies have been pioneered by the institution in Bahrain, including the first Magnetic resonance imaging (MRI) compatible pacemaker (2011); the first implantable loop recorder (2012); the first MRI compatible ICD (2014); and the first cryoablation program for atrial fibrillation (2016). In 2012, a 3D mapping system was established which utilizes the ensite velocity to diagnose and manage complex arrhythmias to ensure optimal patient care. Currently, ensite precision has been upgraded from ensite velocity with some ablation procedures carried out with zero fluoroscopy. For more complex ablations procedures, the latest CARTO 3D mapping system was acquired recently. Moreover, a remote monitoring program for implantable devices was also launched in 2018 to enable patients to improve their follow up from the comfort of their own homes.

Based on Bahrain's population, this EP lab is one of the busiest in the region. Providing expertise in managing various arrhythmias, as well as handling investigations and treatments for patients who survive cardiac arrest or who are at the risk of having this condition, the cardiac EP department aspires to be a leader in the kingdom. Additionally, being the only center on the island that implants and follows up on cardiac implantable electronic devices (CIEDs), our device clinic is completely unique and stands out.

Elective EP procedures, particularly arrhythmia ablation procedures and other non-urgent device implants, were restricted due to Coronavirus disease-2019 (COVID-19). Nonetheless, there has been an increase in pacemaker implants in 2020 when compared to the previous years.

The procedure of implanting pacemakers was previously performed by cardiac surgeons and interventional cardiologists. Approximately 10-12 pacemakers were implanted per year, mostly singlechamber pacemakers. This number has increased dramatically since 2008. Now, all pacemakers, ICDs and CRTs implanted are compatible with MRIs.

For electrophysiology studies, Mohammed bin Khalifa bin Salman Al Khalifa Cardiac Specialist Centre is performing various forms of ablations of supraventricular tachycardias and normal heart ventricular tachycardia (VT) including premature ventricular beats (VPBs).

A total of 12 arrhythmia ablations were conducted around 2010 which grew to more than 80 per year. The average volume of low voltage device implants is 70-90 per year, high voltage devices are 50-70 per year, loop recorders are 10-15 per year, and arrhythmia ablation procedures are 70-90 per year. Every year, over 1,500 patients with various device 
implants are followed in the device clinic. All procedures are free of charge for Bahraini citizens and expats working in government sectors. The Mohammed Bin Khalifa Bin Salman Al Khalifa Cardiac Centre has hosted EP workshops with international faculty and encouraged academic exchanges with various international institutions. In addition, the department is very active in research and academic activities on a regional and international level.

\section{Funding}

Nil

\section{Conflict of Interest}

Nil 